\title{
Rotation and magnetic activity of oscillating solar-like stars with the Kepler mission
}

\author{
Savita Mathur ${ }^{1, \star}$, Lauren Balliet ${ }^{1,2}$, Rafael A. García ${ }^{3}$, and David Salabert ${ }^{3}$ \\ ${ }^{1}$ Space Science Institute, 4750 Walnut street Suite\#205, Boulder, CO 80301, USA \\ ${ }^{2}$ Clarion University of Pennsylvania, 840 Wood Street, Clarion, PA 16214, USA \\ ${ }^{3}$ Laboratoire AIM, CEA/DRF-CNRS-Université Paris Diderot; IRFU/SAp, Centre de Saclay, 91191 Gif-sur- \\ Yvette Cedex, France
}

\begin{abstract}
For the last few decades the investigation of stellar magnetic activity has been conducted through spectroscopic and spectropolarimetric surveys. This led not only to the detection of magnetic cycles in other stars but also to variable and magnetic activity. For the Sun, the magnetic activity is described as the interaction between convection, rotation, and magnetic field. To study magnetic activity of solar-like stars we need to have the knowledge of the surface rotation period, the properties of magnetic activity, and the structure of the stars. We present the results obtained from the studies of Kepler solarlike targets in terms of rotation periods, magnetic activity proxies and magnetic activity cycles detected. We can then combine this information with asteroseismic studies to have a broader picture of stellar magnetic activity.
\end{abstract}

\section{Introduction}

The Kepler mission has observed more than around 200,000 stars. Recently, a new catalog of the Kepler star properties was released for 197,096 targets observed providing for instance surface gravity, effective temperature, radius, but also distance and extinction ([1]). This catalog was based on isochrone fitting using inputs from asteroseismology, spectroscopy, photometry along with a Bayesian method. While all these properties are provided on the exoplanet archive, they have been used by the latest planet transit search pipeline to characterize all the planets and planet candidates discovered by the mission, in particular the ones in the habitability zone of the host star. However, stellar magnetic activity is not taken into account in the habitability zone. Indeed, the magnetic activity of the host stars could wipe away the atmosphere of the planet. The study of magnetic activity of solar-like stars also allows us to have a better understanding of the magnetic activity of the Sun.

\section{Analyzing photometric data}

1. Rotation and magnetic activity of solar-like pulsating stars: Different methods can be used to measure surface rotation periods $\left(P_{\text {rot }}\right)$ : periodogram, time-frequency analysis (wavelets; e.g., [2]) as shown in Figure 1, and auto-correlation function (ACF; e.g., [3]).

^smathur@spacescience.org 
We define a photometric magnetic activity index, $<\mathrm{Sph}>$. We compute the standard deviation of subseries of length $5 \times P_{\text {rot }}$ that gives $\mathrm{Sph}(t)$ ([4]). Its mean value provides the $<\mathrm{Sph}>$ index.

We analyzed 540 stars showing solar-like oscillations observed by Kepler ([5]). We obtained a reliable rotation period for 310 stars using the light curves calibrated by [6]. We found that hot stars rotate faster and the photospheric magnetic activity of the pulsating solar-like stars is compatible with the solar magnetic activity during the full cycle, which is also the case for 18 solar analogs in the sample ([7]). This also allowed us to study gyrochronology relationships ([8]).
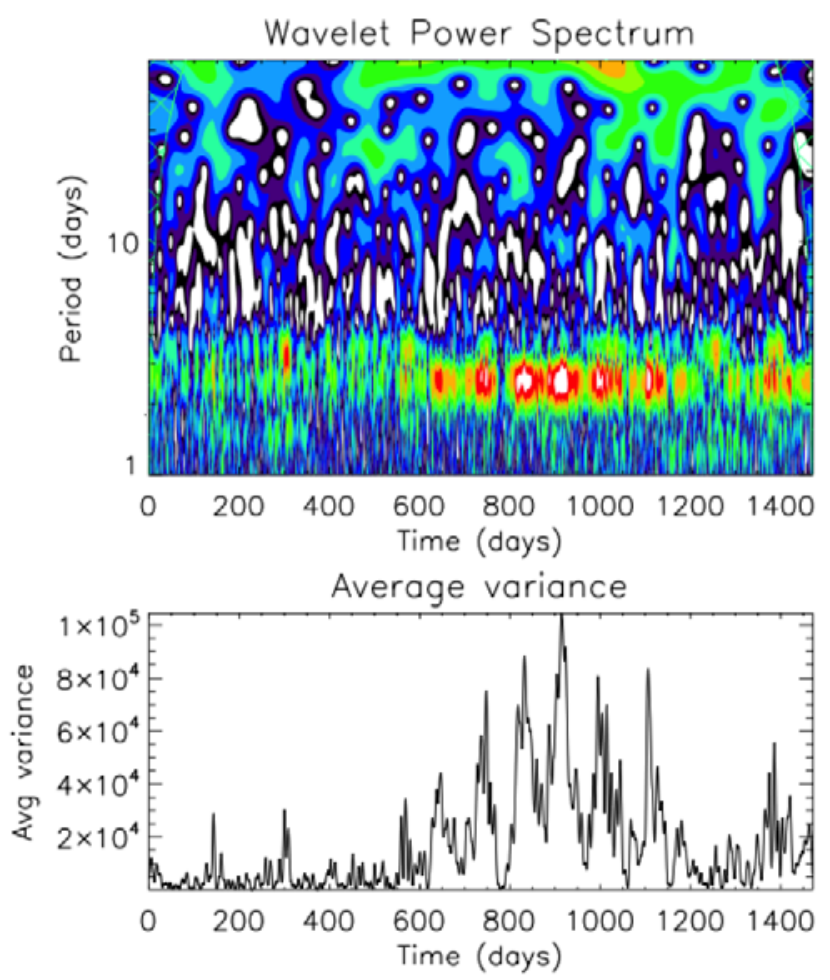

Figure 1. Wavelet analysis of the Kepler target KIC 3733735. Upper panel: wavelet power spectrum resulting from the correlation between the time series and a mother wavelet that we slide along time (x-axis) and change the period (y-axis). Red and white areas correspond to higher power. Bottom panel: projection of the wavelet power spectrum on the $\mathrm{x}$-axis showing the change in the magnetic activity with time.

2. Magnetic Activity Cycles: Time-frequency analysis allows us to study possible magnetic activity cycles ([9]). Figure 1 shows an example of a cycle observed for an F-type star.

Acoustic modes are also known to be affected by magnetic activity ([10]). During maximum activity the amplitudes of the modes decrease while the frequencies increase. This has been observed on the Sun and a few solar-like stars targeted by CoRoT and Kepler (e.g., [11, 12]). 
Acknowledgments: SM would like to acknowledge support from NASA grants NNX12AE17G, NNX14AB92G and NNX15AF13G and NSF grant AST-1411685. LB acknowledges NSF REU grant 1157020. DS and RAG acknowledge the financial support from the CNES GOLF and PLATO grants.

\section{References}

[1] Mathur, S., Huber, D., Batalha, N. M., et al., ApJS, 229, 30 (2017)

[2] Mathur, S., García, R. A., Régulo, C., et al., A\&A, 511, A46 (2010)

[3] Ceillier, T., van Saders, J., García, R. A., et al., MNRAS, 456, 119 (2016)

[4] Mathur, S., Salabert, D., García, R. A., \& Ceillier, T., JSWSC, 4, A15 (2014)

[5] García, R. A., Ceillier, T., Salabert, D., et al., A\&A, 572, A34 (2014)

[6] García, R. A., Hekker, S., Stello, D., et al., MNRAS, 414, L6 (2011)

[7] Salabert, D., García, R. A., Beck, P. G., et al., A\&A, 596, A31 (2016)

[8] van Saders, J. L., Ceillier, T., Metcalfe, T. S., et al., Nature, 529, 181 (2016)

[9] Mathur, S., García, R. A., Ballot, J., et al., A\&A, 562, A124 (2014)

[10] García, R. A., Mathur, S., Salabert, D., et al., Science, 329, 1032 (2010)

[11] Salabert, D., Régulo, C., García, R. A., et al., A\&A, 589, A118 (2016)

[12] Kiefer, R., Schad, A., Davies, G., \& Roth, M., A\&A, 598, A77 (2017) 